\title{
Tissue-Engineering Strategies to Repair Chondral and Osteochondral Tissue in Osteoarthritis: Use of Mesenchymal Stem Cells
}

\author{
Susanne Grässel • Julia Lorenz
}

Published online: 3 September 2014

(C) The Author(s) 2014. This article is published with open access at Springerlink.com

\begin{abstract}
Focal chondral or osteochondral lesions can be painful and disabling because they have insufficient intrinsic repair potential, and constitute one of the major extrinsic risk factors for osteoarthritis (OA). Attention has, therefore, been paid to regenerative therapeutic procedures for the early treatment of cartilaginous defects. Current treatments for OA are not regenerative and have little effect on the progressive degeneration of joint tissue. One major reason for this underrepresentation of regenerative therapy is that approaches to treating OA with cell-based strategies have to take into consideration the larger sizes of the defects, as compared with isolated focal articular-cartilage defects, and the underlying disease process. Here, we review current treatment strategies using mesenchymal stem cells (MSCs) for chondral and osteochondral tissue repair in trauma and OA-affected joints. We discuss tissue-engineering approaches, in preclinical large-animal models and clinical studies in humans, which use crude bone-marrow aspirates and MSCs from different tissue sources in combination with bioactive agents and materials.
\end{abstract}

Keywords Osteoarthritis - Trauma - Cartilage - Focal defect . Clinical · Preclinical · Animal model · Ovine $\cdot$ Porcine .

Equine $\cdot$ Chondral $\cdot$ Osteochondral $\cdot$ Hyaline $\cdot$ Mesenchymal stem cells $\cdot$ Platelet-rich plasma $\cdot$ Bone-marrow concentrate $\cdot$ Tissue engineering $\cdot$ Collagen $\cdot$ Fibrin gel

This article is part of the Topical Collection on Osteoarthritis

\section{S. Grässel · J. Lorenz}

Experimental Orthopedics, Centre for Medical Biotechnology, BioPark 1, Department of Orthopedic Surgery, University Hospital Regensburg, Regensburg, Germany

\section{S. Grässel $(\bowtie)$}

Orthopedic Surgery, Exp. Orthopedics, University of Regensburg, ZMB / BioPark 1, Josef-Engert-Str. 9, 93053 Regensburg, Germany e-mail: susanne.graessel@klinik.uni-regensburg.de

\section{Introduction}

Focal chondral or osteochondral lesions can be painful and disabling, and predispose patients to osteoarthritis (OA). For long-term repair and regeneration of these defects, cells alone or in combination with biomaterials are implanted at the site of injury. However, not much attention has been paid to microenvironmental effects of the neighboring cartilage and subchondral bone. This is particularly evident in diseases affecting diarthrodial joints, including OA, which is an agerelated and/or trauma-induced multi-factorial, slowly progressing, and primarily non-inflammatory degenerative disorder of the synovial joints culminating in the irreversible destruction of the articular cartilage. As well as metabolic imbalance, activation of the whole endochondral-ossification program, starting with cell proliferation through articularchondrocyte hypertrophy and apoptosis, has been identified as an important determinant of OA progression [1-4].

Articular cartilage lesions greater than $5 \mathrm{~mm}^{2}$ do not heal spontaneously [5], and for therapy it must be kept in mind that cartilage defects are multifactorial and site-specific and thus need both a clear analysis of the underlying pathology and individualized therapy. Chondral or osteochondral lesions of any type are found in $61 \%$ of patients with joint pain and are the most prevalent indication for surgical cartilage repair. The incidence of severe International Cartilage Repair Society (ICRS) grade III lesions and of full chondral ICRS grade IV defects in knee joints are approximately $40 \%$ and $19 \%$, respectively [6]. If left untreated they lead, after a long asymptomatic interval, to full clinical OA. It has been suggested that cartilage repair surgery may be most suitable for patients younger than 40-50 years. Attention, therefore, has been paid to therapeutic procedures for the early treatment of cartilaginous defects. The advantage of local defects is that they are contained within healthy cartilage and bone, and it is likely that delivery of specific growth factors and other 
chondroprotective factors to defect sites will support cartilage healing. Early treatment of cartilaginous lesions could indeed be crucial to slowing down the chronic development of OA. The major challenges in regenerative medicine for cartilage are restoration of a biomechanically competent extracellular matrix (ECM) and intimate integration of this newly synthesized matrix within the resident tissue. To address this specific challenge, autologous chondrocyte implantation (ACI) was developed and has prepared the way for novel cell-based therapy and biomaterial-assisted cartilage engineering [7].

Current treatments for $\mathrm{OA}$ are not regenerative and have little effect on the progressive degeneration of joint tissue. Clinical interventions are primarily symptomatic, with a focus on pain reduction and control of inflammation using nonsteroidal anti-inflammatory drugs and, ultimately, total joint replacement $[8,9]$. One major reason for this underrepresentation of regenerative therapy is that approaches for treating OA with cell-based strategies have to take into consideration both the larger sizes of the defects and the underlying disease process. Fragile neocartilage constructs produced by implanted or injected mesenchymal stem cells (MSCs) or chondrocytes may undergo rapid degradation when situated in inflamed or diseased joints. Therefore the underlying pathology must be brought effectively under control, because otherwise any cell-based treatment strategy of OA is unlikely to be successful long-term. This knowledge implies that cartilage repair lacks a one-for-all therapy.

Here, we present up-to-date treatment strategies using MSCs for chondral and osteochondral tissue repair in posttraumatic and OA-affected joints. We have reviewed current literature for tissue-engineering approaches in preclinical large-animal models and clinical studies of humans using crude bone-marrow concentrate (BMC) and MSCs from different tissue sources in combination with other agents. Figure 1 gives an overview of preclinical and clinical studies referred to in this review.

\section{Clinical-Application Techniques for Autologous BMC and/or $\mathrm{MSC}$}

In principal, two strategies exist for the clinical application of cultured and expanded cells (autologous chondrocytes or MSCs) or cell suspensions (autologous bone-marrow concentrates or whole blood): surgical treatment and injection. Surgical delivery of cells includes transplantation or implantation of cells into the chondral and osteochondral defects. The advantage of this approach is the direct and targeted application on the lesion site; the disadvantage is the invasiveness of the approach, which requires opening of the joint cavity. Intraarticular injections are less invasive, which makes application easier; however, there is no precise way of delivering the cells to the defect site. Depending on the application method, the suspension can be injected directly into the diseased tissue, where the cells eventually populate the target site and stimulate repair via autocrine or paracrine pathways. With respect to human therapy, injection of whole blood or bone marrow, or concentrates thereof, can usually be performed in the operating theatre without major regulatory obstacles. The administration of cell preparations expanded ex vivo requires strict compliance with good medical practice (GMP) and nation-specific requirements.

Bone-marrow concentrate has attracted much interest from orthopedic surgeons as a third-generation intra-articular orthobiological injectable therapy for cartilage disease. Bone-marrow concentrate contains MSC, hematopoietic stem cells, platelets (containing growth factors), and cytokines. The anti-inflammatory and immunomodulatory properties of bone-marrow-derived mesenchymal stem cells (BMSC) or adipose-derived stem cells (ADSC) can facilitate regeneration of tissue. Although marrow suspensions are believed to contain a higher percentage of stromal cells than peripheral blood, both are crude mixtures with low overall abundance of MSCs and undefined mixtures of proteins. The MSC density can be increased by centrifugation of whole blood or marrow, recovery from cell filters, clotting, or other concentration methods. These methods are relatively simple, making them appealing for single-step usage procedures in the operating theatre, and, therefore, are not associated with major regulatory obstacles.

Alternatively cells, and in particular MSCs, can be harvested from blood, bone marrow, or, after digestion, other tissues via their adherence to plastic surfaces. Such cell populations are usually maintained in tissue culture for amplification under controlled GMP conditions using autologous serum. This method offers the opportunity for close monitoring of the cells regarding safety and quality aspects, and for further cell selection. However, this clinical approach has the greatest regulatory obstacles in the specific requirements of each country and continent (European Medicines Agency and U.S. Food and Drug Administration). The use of unprocessed and exvivo-processed cells might be enhanced and supplemented by the use of biomaterial scaffolds, soluble growth factors, nucleic acids, or mechanical stimulation. Additional specific regulatory requirements must be met for the use of each of these supplements and for their combined usage, indicating the complexity of such approaches from a biological, medical, and regulatory perspective (reviewed in Refs. [10, 11•]).

Treatment of Chondral and/or Osteochondral Lesions with MSC in Preclinical Studies Using Large-Animal Models

The average human articular-cartilage thickness is $2.2-$ $2.5 \mathrm{~mm}$ [12] and the average defect size is approximately $550 \mathrm{~mm}^{3}[6,13]$, with $95 \%$ of defects involving cartilage only and not affecting subchondral bone [6, 14]. For testing cell-based treatments, a perfect animal model should mimic as precisely as possible the human defect and articular-cartilage 


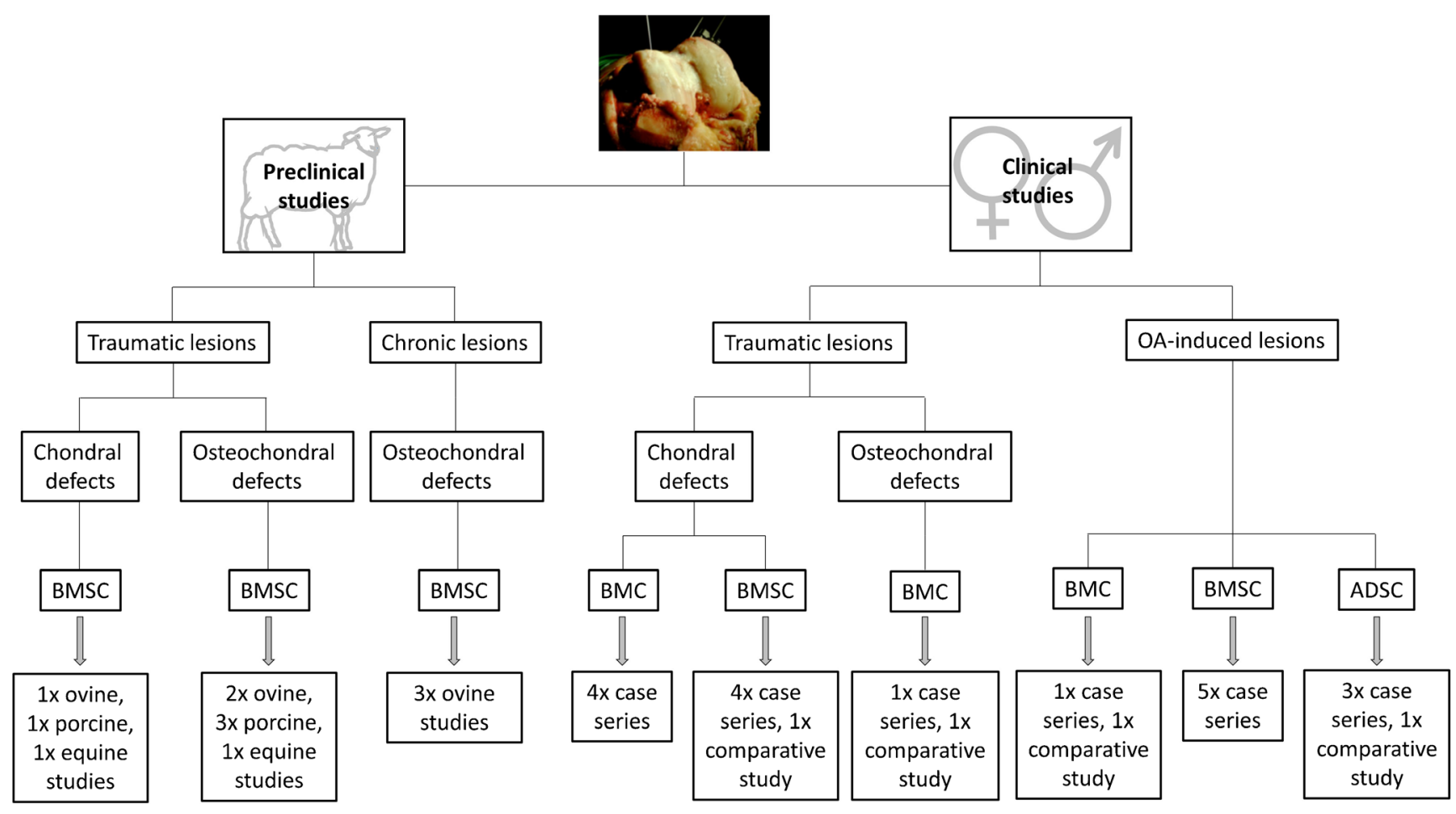

Fig. 1 Overview of preclinical and clinical studies of treatment of traumatic and osteoarthritis-induced defects with mesenchymal stem cells (MSCs) discussed in this review. ADSC $=$ adipose-tissue-derived stem

morphology. A variety of animal models for cartilage lesions are described in literature, ranging from murine, laprine, canine, ovine, porcine, and caprine to equine models (reviewed in Ref. [14]). Small-animal models, for example mouse and rabbit, are often used as proof-of-concept, but articular cartilage is thinner and defects are much smaller compared with humans; in addition, most defects cannot be set without penetrating the subchondral bone plate. Therefore, especially for preclinical studies, large animals including swine, sheep, or horse are more appropriate for modelling human articularcartilage defects and testing the regenerative capacity of cellbased treatments. The following section presents a comprehensive overview of published studies on MSC-based treatment for regenerating localized chondral and osteochondral defects in ovine, porcine, and equine animal models. Table 1 summarizes conditions and treatment outcomes.

\section{Ovine Models}

Sheep are commonly used as animal models for chondral and osteochondral defects, because these animals are readily available, easy to handle, and relatively inexpensive. In addition, the anatomy of the knee joint is similar to that of humans and "second look" arthroscopy is possible. Furthermore, the ovine medial femoral condyle cartilage thickness of $0.4-1.7 \mathrm{~mm}$ $[12,27]$ - although thinner than that of humans - enables production of partial and full-thickness defects that do not cells, $\mathrm{BMC}=$ bone-marrow concentrate, $\mathrm{BMSC}=$ bone-marrow-derived mesenchymal stem cells

penetrate the subchondral bone plate [14]. In osteochondral defects the bone marrow is opened, and one likely situation is that endogenous BMSCs migrate from the bone marrow into the defect, thus contributing to the repair tissue in a manner comparable to the BMSC implant. This setting makes it impossible to determine whether implanted cells or endogenous cells are responsible for defect healing. To circumvent this situation, one ovine study conducted by Mrugala et al. [15] implanted autologous ovine BMSCs into a partial-thickness-cartilage-defect model. BMSCs in passage 1 were mixed with blood and/or chitosan scaffold and/or TGF- $\beta 3$, and the clots generated were filled into cartilage defects set in the internal groove of the patella. The authors observed that BMSCs or the scaffold alone had poor ability to repair these defects, whereas BMSC in the presence of TGF- $\beta 3$ and chitosan-based-scaffold-generated tissue was positive for aggrecan and collagen II, indicating hyaline-like repair tissue. After two months the defects were filled with this cartilagelike tissue; this tissue was only moderately integrated with the surrounding cartilage, indicating that investigations at later time points are required to observe full integration of newly formed tissue occurring after several months (Table 1).

For BMSC treatment of ovine osteochondral defects, five studies have been reported. A novel, promising approach seems to be the application of BMSCs that have been predifferentiated ex vivo to the chondrogenic stage; this strategy was tested in three studies by the same group using 


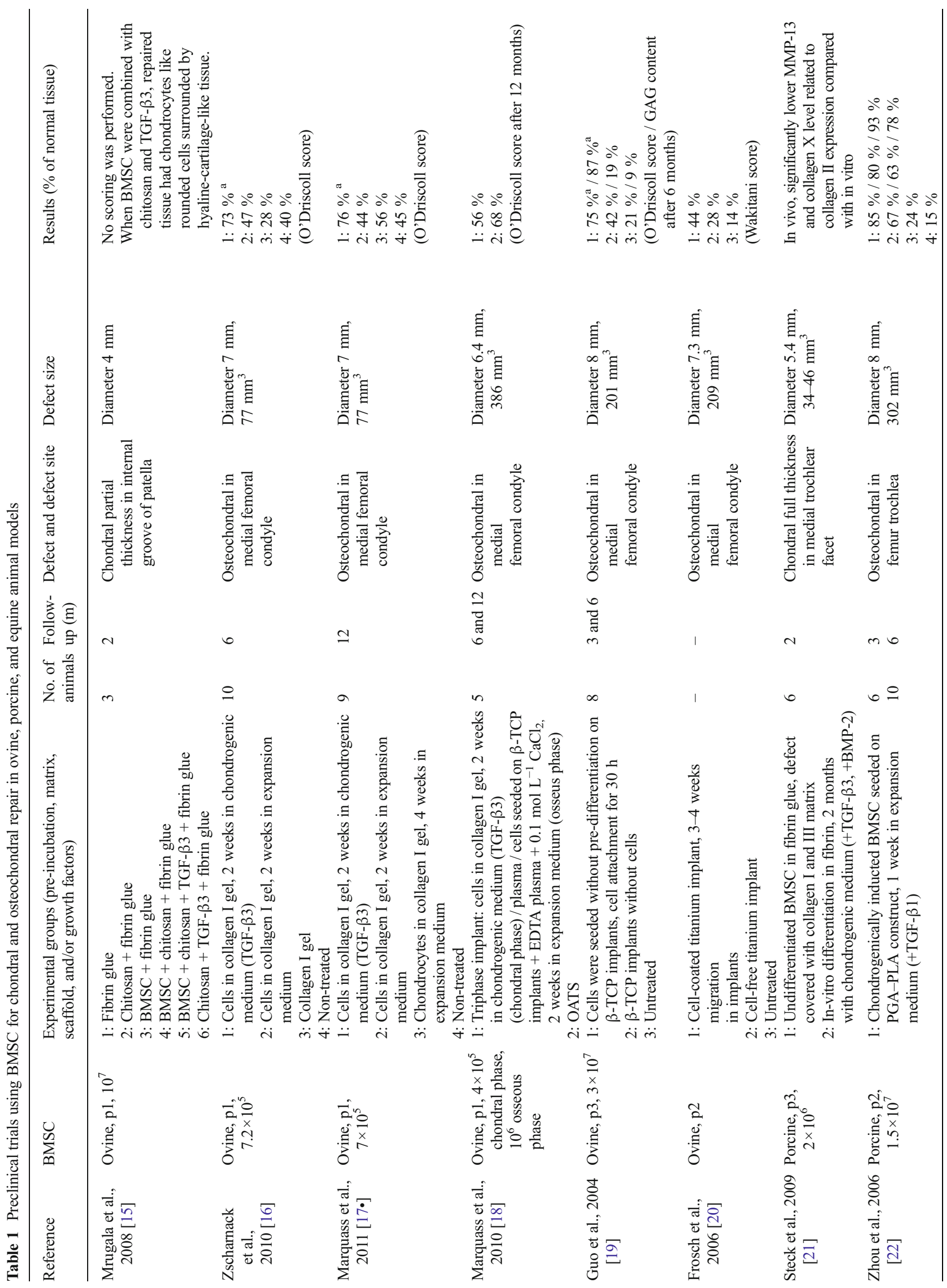




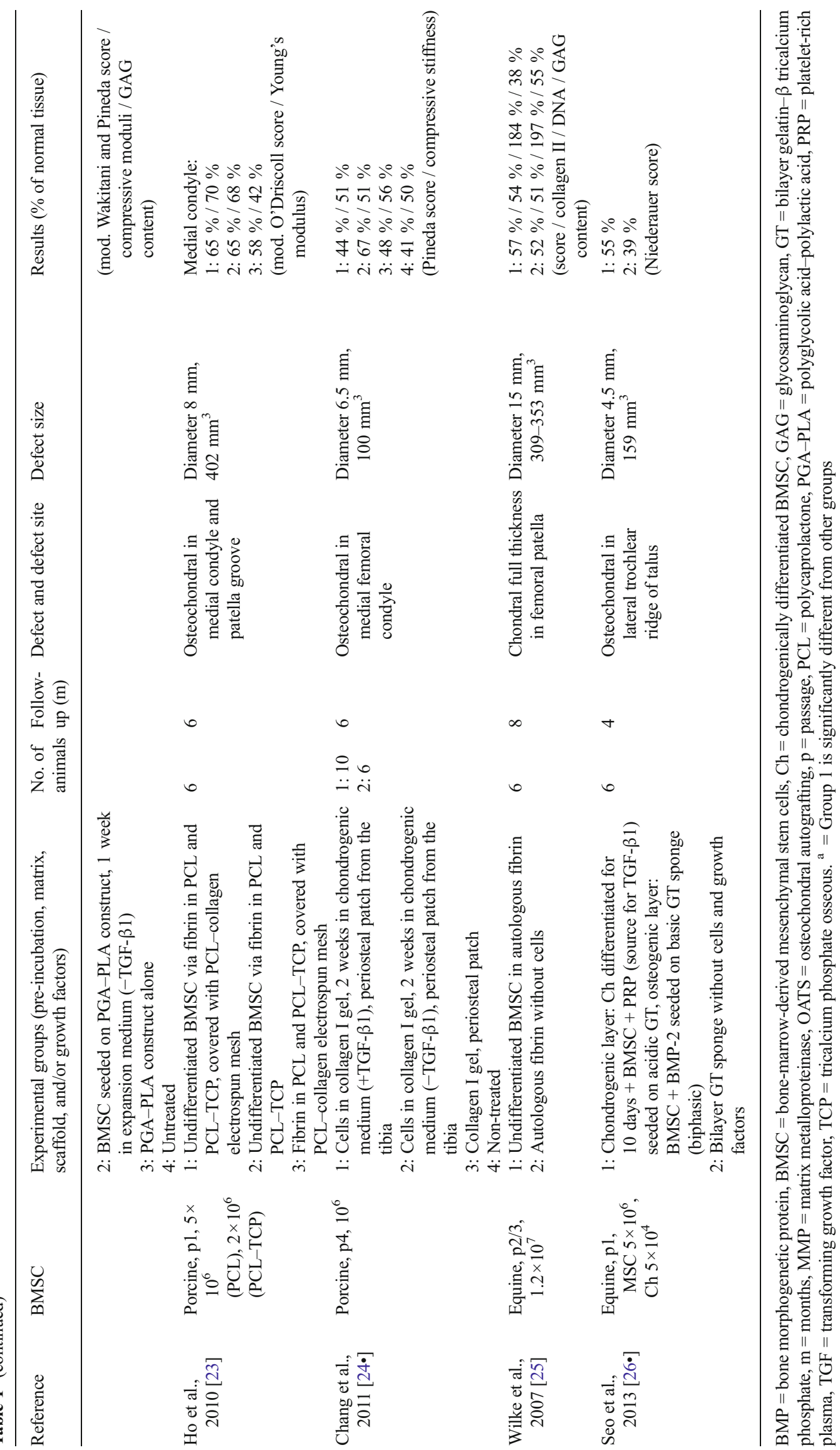


comparable experimental setups $[16,17 \bullet, 18]$. A unique aspect of these three studies was that the treatment was performed six weeks after defect setting, whereas in all other large-animal studies defects were treated directly after setting. Delaying treatment after defect setting creates a clinically relevant situation that resembles chronic post-traumatic human OA. In the first study by Zscharnack et al. [16], predifferentiated BMSCs achieved better regenerative results after six months, as measured by gross appearance and O'Driscoll [28] and ICRS [29] scores with respect to collagen II content and hyaline-cartilage appearance, than undifferentiated BMSCs, collagen I gels alone, and untreated controls. However, integration of newly formed cartilage-like tissue was incomplete at six months, and the important question of whether the BMSCs had adopted a stable chondrogenic phenotype, without further progression to hypertrophy at time points beyond six months, was not addressed. In the second study, Marquass et al. [17•] compared the regenerative capacity of ex-vivo-pre-differentiated BMSCs with differentiated autologous articular chondrocytes after 12 months, and found higher O'Driscoll [28] and ICRS scores with predifferentiated BMSCs in collagen I gels than with chondrocytes in MACT gels, undifferentiated BMSCs in gels, and untreated controls. Furthermore, repair tissue generated by pre-differentiated BMSCs had no sign of degradation within one year. In this line, the approach was enhanced by embedding pre-differentiated BMSCs into triphasic constructs [18]. These triphasic constructs were compared with standard osteochondral autografting (OATS), again using an ovine osteochondral-defect model. At six months no significant difference in histological scores between the two groups was detected. At this time point the triphasic-construct group had superior cartilage bonding, whereas at 12 months the OATS group had superior cartilage-matrix morphology. Because of an observed sinking of the triphasic constructs, and a high variability in the quality of the repaired tissue, the authors did not believe the triphasic construct superior to OATS. A study from Guo et al. [19] used BMSCs without pre-differentiation seeded on $\beta$-tricalcium phosphate (TCP) implants for treatment of osteochondral defects. After six months, hyaline-like tissue covered the surfaces of the defects and a perfect interface between engineered tissue, the adjacent cartilage, and underlying bone was observed, whereas in control groups ( $\beta$-TCP scaffold and untreated) the defects were clearly visible and incompletely repaired. The authors concluded that undifferentiated MSCs on $\beta$-TCP scaffolds have promising potential for clinical application. The last ovine study analyzed the regenerative capacity of undifferentiated BMSCs seeded in titanium implants into large osteochondral defects. After six months, Frosch et al. [20] found that $50 \%$ of BMSCcoated implants were osseo-integrated, with complete regeneration of subchondral bone; the regenerated cartilage contained collagen I and collagen II, indicating hyaline-like- cartilage regeneration. In contrast, the other $50 \%$ of the BMSC-coated implants, the cell-free implants, and the nontreated controls had incomplete healing, osseo-integration failure, and formation of fibrocartilage instead of hyaline cartilage (Table 1).

\section{Porcine Models}

Pigs are not commonly used for cartilage-defect research, because of handling difficulties and logistical requirements regarding housing. A point in favor of using pigs is that the articular cartilage thickness of $1.5-2.0 \mathrm{~mm}[12,30,31]$ is more similar to humans than that of ovine cartilage and enables easier production of partial and full-thickness defects without penetrating the subchondral bone plate [14], better reflecting human articular-cartilage defects.

Proof of a stable in-vivo cartilaginous-tissue phenotype of implanted BMSCs is the focus of a study by Steck et al. [21]. The group analyzed expression of collagens II and X and of MMP-13 to compare the performance of BMSCs predifferentiated in vitro to a chondrogenic stage versus undifferentiated BMSCs when implanted into minipigs with acute, full-thickness cartilage defects on the trochlear ridge of the femur. Defects were filled with the undifferentiated BMSCs and sealed with a collagen I and III matrix and commercial fibrin glue. Follow-up times for assessment of morphological and molecular tissue aspects were up to eight weeks. The data revealed that, in comparison to the pre-differentiated BMSCs, the application of undifferentiated BMSC in the orthotopic environment resulted in lower COL10A1/COL2A1 and MMP13/COL2A1 mRNA ratios. The reason for the discrepancy between in-vitro and in-vivo results is poorly understood, and the authors speculated that unknown signaling molecules and biomechanical stimuli derived from neighboring cartilage and/or bone tissue may have an important effect. On the basis of their data, they regarded application of BMSCs pre-differentiated ex vivo as unfavorable for cartilage repair until better in-vitro induction procedures for chondrogenesis become available. In addition, to obtain a repair tissue comparable to healthy cartilage, a study duration of eight weeks is too short.

The regenerative capacity of BMSCs in porcine osteochondral defects was reported in three studies. Zhou et al. [22] analyzed the repair capacity of pre-differentiated and undifferentiated BMSCs in biodegradable PGA-PLA scaffolds. After six months, both groups with cells had better reparative results regarding gross appearance and histology than were obtained for the group with scaffold alone and the untreated group. Repair tissue of pre-differentiated BMSCs had almost normal cartilage and subchondral-bone-like properties. Concerning the question of if and for how long implanted BMSCs remain in the defect, a study revealed that GFP-labeled implanted BMSCs are located in the regenerated 
cartilage-like tissue and subchondral bone even after seven months of follow-up. In a carefully designed and evaluated study, Ho et al. [23] used undifferentiated BMSCs embedded with fibrin in polycaprolactone (PCL) and PCL-TCP scaffolds and covered the defect with PCL-collagen electrospun mesh. In contrast with the findings of Zhou et al., defects were set in weight-bearing areas (medial condyle and patellar groove) of the joint. After six months, BMSCs and PCLcollagen electrospun mesh had a positive effect on morphological outcomes of cartilage-like repair tissue. Notably, healing was inferior at the patellar groove compared with the medial condyle, and this was attributed to site-specific biomechanical features.

Chang et al. [24•] obtained better histological Pineda scores [32] with undifferentiated BMSCs compared with differentiated BMSCs, collagen I matrix alone, or untreated defects in minipigs after six months. However, the quality of the regenerated tissue was inferior and did not differ significantly between experimental and control groups. In contrast with the two previously described studies, osteochondral defects were smaller - which may explain the high degree of spontaneous healing of non-treated defects-when BMSCs were used at higher passages without scaffolds inserted into the defect (Table 1).

\section{Equine Models}

The horse is the largest model available and, having an average medial-femoral-condyle-cartilage thickness of 1.75-2 mm [33], it has even more similarity to human cartilage in thickness than other large-animal models. "Second look" arthroscopy is possible, and partial or full-thickness cartilage defects without penetration of the subchondral bone plate can be set. However, the horse is a companion animal and thus ethical aspects are an important factor. Furthermore, horses are not bred specifically for biomedical research, handling is difficult, and housing is very expensive [14].

In Wilke et al. [25], $15 \mathrm{~mm}$ full-thickness cartilage defects were set in the femoral patella and filled with undifferentiated BMSCs embedded in autologous fibrin. A second-look arthroscopy and biopsy were obtained for each animal after 30 days and animals were euthanized after eight months. Short-term assessment revealed a significant improvement of arthroscopy scores and increased collagen II-containing fibrous tissue in defects treated with BMSCs compared with those treated with fibrin matrix alone. However, long-term assessment revealed no difference in BMSC-treated defects compared with those treated with fibrin matrix alone regarding GAG and/or collagen II content and matrix biochemical assays. For both groups, the repair tissue differed markedly from normal cartilage in cartilage-quality scores and biochemical properties. In this study defect healing might be affected by compromised motion because, after recovery from anesthesia, the horses were housed without exercise for five weeks and then with a daily exercise regimen of a 5 min walk for a further seven weeks.

Seo et al. [26•] analyzed the repair capacity of BMSCs loaded on biphasic sponge scaffolds and implanted in osteochondral defects. The chondrogenic layer consists of a acidic gelatin- $\beta$-tricalcium phosphate (GT) sponge loaded with platelet-rich plasma, BMSCs, and chondrogenically differentiated BMSCs (without TGF- $\beta$ ), whereas the osteogenic layer is composed of a basic GT sponge loaded with BMP-2 and BMSCs. After four months, the test group had significantly higher radiographic, QCT, macroscopic, and histological scores than the control group with biphasic sponge and without cells or growth factors (Table 1).

\section{Comparison to Human Situation}

Despite the fact that $95 \%$ of human defects are of chondral and not osteochondral nature [6, 14], only a few pre-clinical studies $[15,21,25]$ have created isolated chondral defects to analyze the cartilage-regeneration capacity of BMSCs. Furthermore, most studies suffer from too-short follow-up times - usually less than 12 months - which do not enable satisfactory analysis of repair tissue with respect to stability of the chondrogenic phenotype, hyaline-like appearance, defectsite integration, and biomechanical characteristics. For osteochondral defects, all large-animal studies, irrespective of the animal model, reported improvement in regeneration when BMSCs were used for defect repair [16, 17•, 20, 22, 23, 24, 25$]$. A beneficial effect of an additional ex-vivo predifferentiation phase of MSCs is a matter of debate on the basis of current knowledge [16, 17•, 21, 22].

Several aspects remain to be addressed in future studies, including the effect of animal age on the extent of repair of chondral defects at weight-bearing areas. Importantly, only the three ovine studies from the Leipzig group featured a study design with a six-week gap between defect setting and implantation of BMSCs, thereby inducing an early OA stage. In addition, thus far only MSCs isolated from bone marrow have been used. Other MSC sources, for example adipose tissue, might provide many advantages because of easier cell preparation, a higher percentage of MSCs compared with differentiated cells, and better availability. No study has been performed using only BMCs to study the cartilage-regeneration capacity of crude cell suspensions including those used frequently for human treatment (see below). Most importantly, in all studies (except the one from the Richter group [21]) no specific marker for hypertrophic differentiation of the BMSCs was evaluated and therefore no conclusion can be drawn regarding the phenotypic stability of the implanted cells. Furthermore, to find out under which conditions regenerated tissue best reflects normal tissue-like properties, the overall comparability between the study procedures must be 
improved with respect to follow-up times, scoring systems, biomechanical measurements, and biochemical properties.

Human Clinical Studies Using Autologous MSC and BMC for Treatment of Chondral and Osteochondral Lesions: Promising Tissue-Engineering Approaches

Cartilage defects that arise from an underlying disease process (for example $\mathrm{OA}$ ) are distinct from focal cartilage lesions that result from acute injury or osteochondrosis dissecans, and this difference must always be taken into consideration. Specifically, acute cartilage injury and osteochondrosis dissecans often occur in otherwise healthy joints; the patient might be young, and probably only the focal defect will require localized treatment. In contrast, patients with OA are likely to be elderly, and often the entire articulating surface will require treatment. Repair of lesions may provide symptomatic relief and delay the progression of OA symptoms, but without effective treatment of the underlying disease any improvement is likely to be short-lived [34]. Thus, common to all types of cell-based therapy is that they are not recommended for treatment of expanded, arthritic cartilage lesions and kissing lesions. Rather, they are recommended for treatment of local, full-thickness chondral and osteochondral defects, ideally with an intact cartilage interface [35].

\section{Trauma-Induced Chondral and Osteochondral Defects}

Microfracturing If an articular cartilage lesion below $1-4 \mathrm{~cm}^{2}$ is confirmed, the first choice of treatment is often microfracturing (MFX), the penetration of the subchondral bone plate by creating small holes. Bleeding from the subchondral bone spaces yields a blood clot (often called superclot), which is believed capable of stimulating attraction, proliferation, and chondrogenic differentiation of MSCs arising from the bone marrow. The advantage of a marrowstimulating technique is that several of the main objectives of cartilage repair are fulfilled: it is an easy, simple, minimally invasive, low-morbidity, and single-stage procedure, and is a cost-effective technology with few associated complications and a high capacity for creation of durable cartilage-repair tissue that can delay the time to joint replacement [36, 37]. However, clinical results are age-dependent. Marrow stimulation results in improved function and reduced pain for up to $75 \%$ of young patients after five years and up to $80 \%$ of patients after an average of 11 years of follow-up [38, 39]. Repair tissue is mostly fibrocartilage or a hybrid of hyaline and fibrous cartilage, of inferior quality and mechanical stability compared with the original cartilage matrix. Formation of an insufficient cartilage ECM might be promoted by an inadequate microenvironment of the superclot, because its composition is poorly defined. Other limitations are that only partial defect filling is achieved, because of shrinking of the superclot and low incidence of MSCs in the bone-marrow fraction (Reviewed in [37, 40]).

Application of BMC to Trauma-Induced Defects MSC can be used as a cell suspension expanded by culture or from bonemarrow concentrate (BMC). However, these products differ profoundly in composition. The advantage of using BMC over cultured MSCs is that the cells are not passaged, but the disadvantage is that BMC gives a more heterogeneous composition compared with expanded MSC [41•]. Studies have been reported in which BMC, usually combined with other regenerative techniques, is applied to trauma-induced human-cartilage defects. The group of Gigante published three case series in which they treated five patients with isolated femoral condyle lesions by combining BMC with the autologous matrix-induced chondrogenesis (AMIC) technique [42]. In a second study, they described a novel arthroscopy technique that combines MFX, autologous BMC, and a protective collagen-based scaffold [43]. The objective of both procedures is to augment the original single-stage procedure with BMC and to increase defect filling and the rate of hyaline-like-cartilage regeneration. The procedure combining MFX, BMC, and a protective collagen scaffold is inexpensive and reproducible and has obtained regeneration of hyalinelike cartilage. A third study from this group reported on a combination of MFX, BMC, and resorbable polyglycolic acid-hyaluronic acid (PGA-HA) membranes. Nine patients with focal lesions of the condylar articular cartilage were consecutively treated with arthroscopic PGA-HA-covered MFX and BMC. Macroscopic assessment of cartilage after 12 months revealed that one repair attempt appeared normal, three almost normal, and one abnormal. Histological analysis revealed hyaline-like-cartilage-repair-tissue formation in one case and MRI at 8-12 months follow-up revealed complete defect filling [44•]. A comparable approach to treating osteochondral knee and talar dome lesions is reported by the Giannini group, who combined BMC and HA-scaffolds (or collagen powder) with platelet-rich fibrin (PRF) gel in a onestep repair technique and treated 48 patients, with an average follow-up of 24-35 months. Histological evaluation revealed regenerated tissue with a variety of degrees of remodeling irrespective of biomaterial used, although none had entirely hyaline cartilage. These data suggest the one-step technique is a possible alternative for cartilage repair, obtaining improved functional scores and overcoming the disadvantages of previous techniques [45]. In this line, a recent case-series study reported treatment of large cartilage lesions of up to $12 \mathrm{~cm}^{2}$ (ICRS grade III-IV) with BMC, resulting in significant improvement in all clinical scores for 52 out of 54 patients [46]. In a study comparing $\mathrm{BMC}$, open autologous chondrocyte implantation (ACI), and ACI arthroscopy, clinical improvement was similar in all three groups [47] (Table 2). 


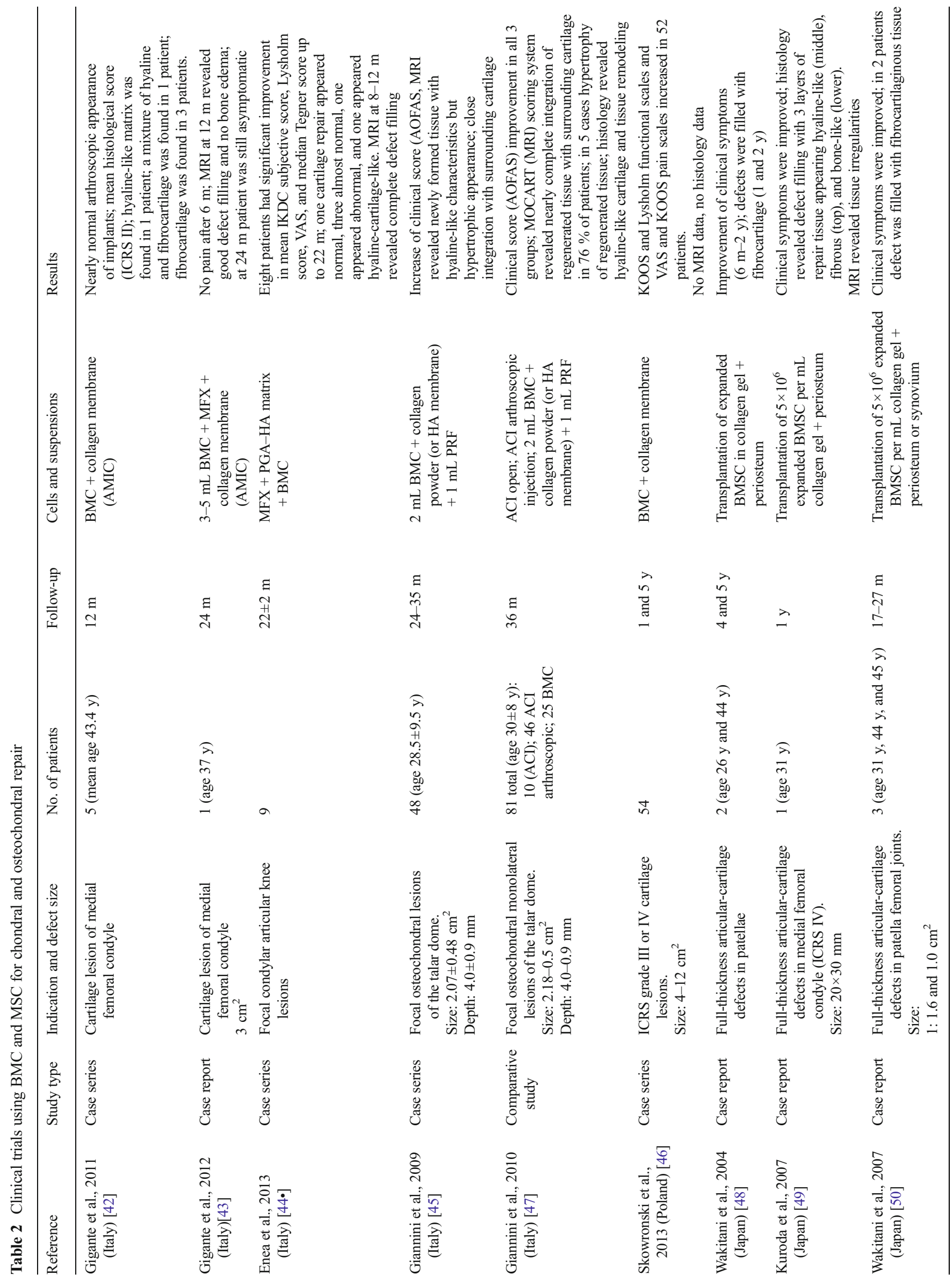




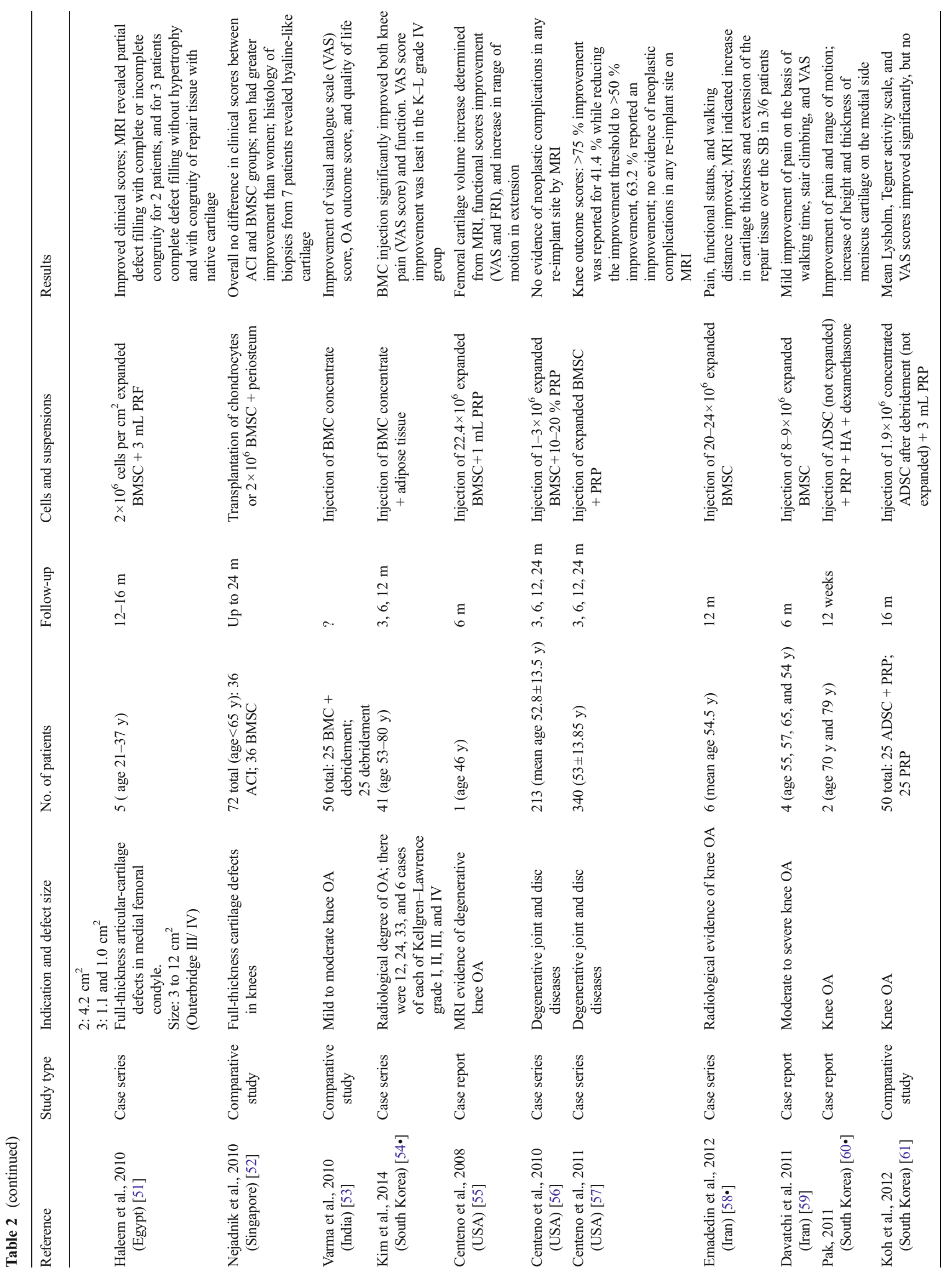




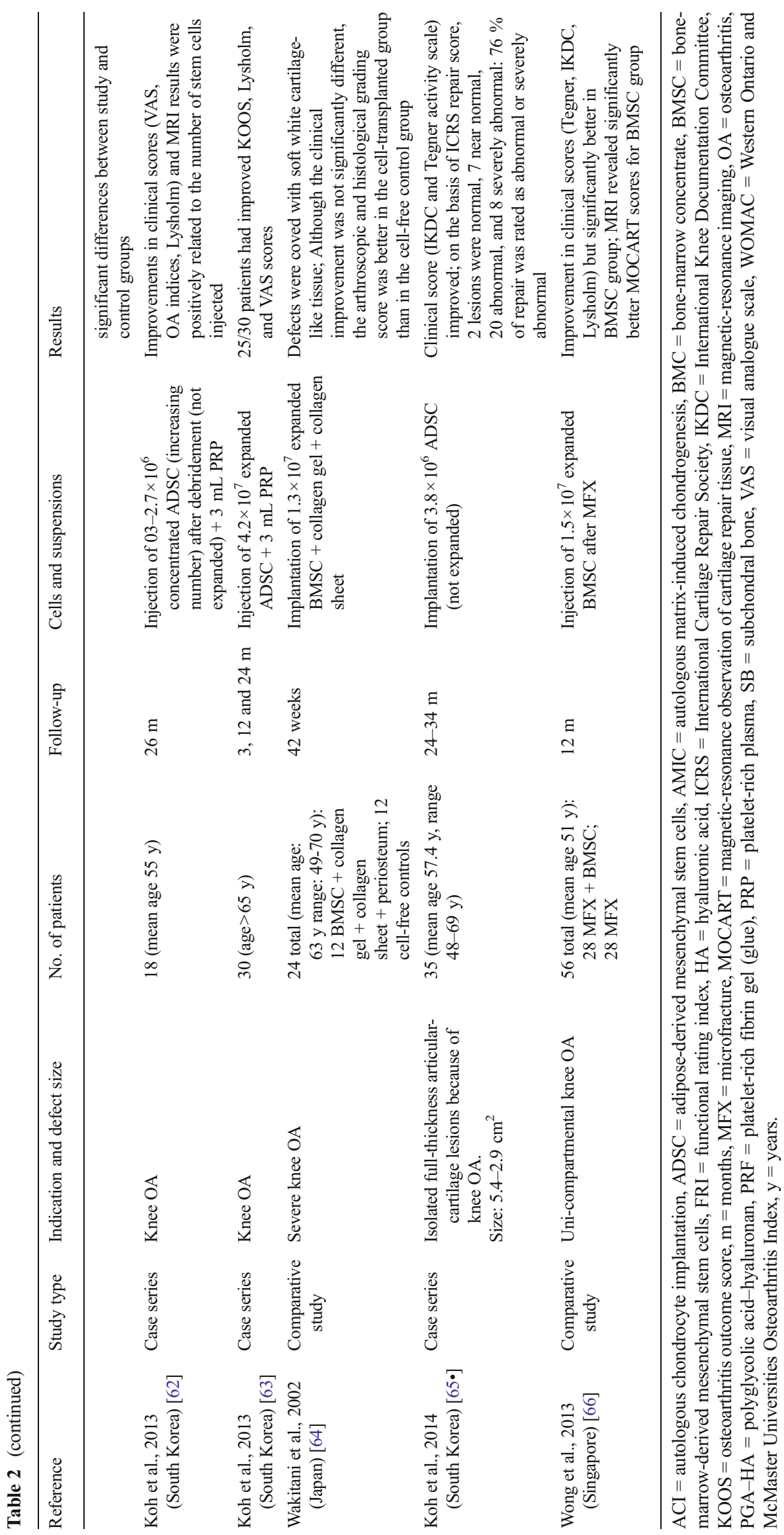


Application of Expanded BMSC to Trauma-Induced Defects The second principal treatment strategy, using expanded BMSC, might be difficult to manage from a legal perspective (because it is regarded as pharmacological drug administration), but positive results have been reported from preclinical animal and clinical human studies. The advantage of expanded MSC is a higher number of cells; the disadvantage is an increased contamination risk during expansion [41•]. The Wakitani group was the first to transplant autologous BMSC, aspirated from the iliac crest and embedded in a collagen gel; these were transplanted into full-thickness patella-femoral-articular-cartilage defects of two patients and covered with a periosteal flap. Although clinical symptoms were improved, histological evaluation 12 months later revealed that the defects were filled with fibrocartilage and not with hyaline-like-cartilage tissue [48]. Three years later the group used the same procedure to implant BMSC into full-thickness cartilage defects of patella-femoral joints. Again, clinical symptoms improved and were maintained throughout the follow-up period. However, it was not possible to unambiguously determine whether the defects were repaired with hyaline cartilage, although biopsies were safranin-O or toluidine-blue positive. Much more likely was that the defects were filled with fibrocartilage-like tissue [49, 50]. In 2010, Haleem et al. transplanted BMSC embedded in platelet-rich fibrin glue (PRF) into full-thickness cartilage defects; compared with the collagen sheet (bovine and porcine origin) PRF has the advantages of being autologous and bio-resorbable. On the basis of MRI, after 12 months the authors concluded that in three out of five patients the defects were filled with tissue resembling native cartilage without signs of hypertrophy [51]. The only comparative study, reported by Nejadnik et al., compared transplantation outcomes for autologous collagen-embedded BMSC versus chondroctyes transplanted into full-thickness knee-cartilage defects in a cohort study. Analysis after an up-to-two-years follow-up period revealed similar functional and clinical results, with the clear advantage that the BMSC treatment required only one operation and minimized donor-site morbidity [52].

These cartilage-repair techniques were able to generate repair tissue which up to a certain point approximates the characteristics of naive hyaline cartilage, but which still more closely represents undesired fibrocartilage tissue. MSC and BMC treatment strategies obtained a marked reduction in procedure, morbidity, and cost by using a "one step" technique able to overcome all the disadvantages of previous repair techniques. However, clinical data on both strategies (i.e. delivering cultured MSC or $\mathrm{BMC}$ to the defect site), although promising, must be regarded as preliminary because most reported studies were single or series case studies without valid and sound controls (Table 2).

\section{OA-Induced Defects}

The above intervention strategies were performed mostly on younger patients without clinical and radiological symptoms of OA. Important for the choice of cell-application strategies is the fact that $\mathrm{OA}$ is a chronic disease which results in multiple cartilage lesions during pathogenesis, whereas trauma in general results in a single localized chondral or osteochondral defect. Therefore, the easiest way to treat OA-mediated lesions would be intraarticular injection of MSC or BMC.

Injection of BMC In a study by Varma et al., 50 patients with mild to moderate knee OA were selected and divided into two groups. One group received arthroscopic debridement alone and the other received buffy coat (mesenchymal-stem-cell concentrate) injection with the arthroscopic debridement. On follow-up, patients were assessed on the basis of visual analogue scale (VAS) score and OA outcome score. The results suggest that injection of BMC much improved the overall OA outcome score, especially the quality of life within and at the end of the follow-up period [53]. A more recent study by Kim et al. used combined intraarticular injection of autologous BMC and adipose tissue (BMAC) into the knees of 41 patients with radiographically assessed OA (assessed on the basis of the Kellgren-Lawrence $(\mathrm{K}-\mathrm{L})$ grade I-IV scale). BMAC injection significantly improved both knee pain and function in all patients. The authors suggest that the injection would be even more effective in early to moderate phases of OA [54•] (Table 2).

Injection of BMSCs and ADMSCS There are several case reports in which BMSCs expanded in vitro were injected into OA joints. The approach of Centeno et al. [55] is interesting, because they augmented the single BMSC injection with autologous platelet lysates prepared from platelet-rich-plasma (PRP) aspirates. The pre and postprocedure MRI analysis revealed increased meniscus and cartilage volume, and at three-month follow-up the modified VAS scores decreased by $95 \%$. Two subsequent uncontrolled studies from the same group using the same treatment procedure were conducted on a large group of patients suffering from OA and other intraarticular pathology $[56,57]$. However, only approximately $60 \%$ of the patients had knee improvement scores thereafter. In this line, suboptimal results were reported after a single injection of expanded and partially characterized BMSCs. This study was a phase-one clinical trial which recruited six patients with radiological evidence of knee OA requiring joint-replacement surgery. Pain, functional status of the knee, and walking distance tended to improve up to six months post-injection, after which pain seemed to be slightly increased and the walking ability of the patients slightly decreased. Comparison of MRI at baseline and six 
months after the stem-cell injection revealed increased cartilage thickness, increased extension of the repair tissue over the subchondral bone, and much-reduced size of subchondral bone-marrow lesions in three out of six patients [58•]. Similar results were obtained in a second uncontrolled pilot study [59].

Of much clinical interest would be the use of ADSCs obtained from lipoaspirates, which offer several advantages over BMSCs when used for cartilage-tissue engineering. ADSCs are more abundant and easily available, and they confer a similar potential to differentiate and to direct formation of cartilage-like tissue. In 2011, Pak et al. [60•] reported good results after treatment of two patients affected by knee OA with the injection of concentrated ADSCs (not expanded or cultured) together with hyaluronic acid (HA), dexamethasone, and PRP. Concentrated ADSCs were obtained by double centrifugation of lipoaspirates and digestion with collagenase. After three months subjective pain and functional status improved, and MRI revealed significantly increased cartilage thickness [60•]. This was followed by three studies from Koh et al. [61], who used concentrated ADSCs derived from the infrapatellar fatpad for treatment of knee OA. First, they presented a case-control study with a total of 25 patients, who each received a single injection of ADSCs and, subsequently, PRP [61]. Clinical results were promising, and the group conducted two further case studies (not controlled) with a similar treatment procedure, but with a different source of ADSCs in the second study. All applied clinical outcome and MRI cartilage scores improved significantly [62, 63]. These studies suggest that ADSC therapy for knee OA is effective in promoting cartilage healing, reducing pain, and improving function, and therefore seems to be a promising option for OA treatment in elderly patients (Table 2).

Implantation of BMSCs and ADSCS The first report describing the implantation of expanded BMSCs into OA knee lesions also came from the Wakitani group. They conducted a comparative study on patients who had undergone high tibial osteotomy (HTO) because of OA. Twelve patients received transplantation with expanded autologous BMSCs, which were embedded in a gel composed of type I collagen and implanted as a collagen sheet, and 12 patients served as cell-free controls receiving the collagen sheet alone. The BMSC-collagen construct was positioned into a large defect of the medial femoral condyle, where cartilage was lost and subchondral bone was exposed, and covered with a periosteal flap. Approximately a year later clinical scores did not differ between the groups, but arthroscopy and histological scores were better in the cell-transplanted group [64]. In a recent report from the Koh group, ADSCs were implanted into an isolated full-thickness cartilage lesion in OA knees. Patients with multiple cartilage lesions were excluded, as were those with a history of marrow-stimulation procedures, i.e. MFX or subchondral drilling. Directly after isolation from fat tissue, cells were dripped into the cartilage lesion and the knee was held in a stationary position for $10 \mathrm{~min}$. No supporting biomaterial was used, nor were the cells kept in position by covering with a matrix or periosteal flap, but simple adherence to the subchondral bone was permitted. Although the authors suggested that MSC implantation could have great potential for treating OA lesions, the second-look arthroscopy findings revealed that in $76 \%$ of the patients the repair was abnormal or severely abnormal by ICRS standards. They concluded that the development of an advanced surgical procedure with tissue-engineered scaffolds may be needed to treat patients with OA-induced large cartilage lesions [65•].

In general, it is not recommended that the MFX technique alone should be used for treatment of OA-mediated degenerative cartilage lesions. However, in a recent randomized, controlled clinical study, MFX combined with intraarticular injection of BMSCs was used to treat 28 patients with unicompartmental OA knees undergoing HTO, whereas the second group of 28 patients received an intraarticular HA injection instead. Assessment of IKDC and MOCART scores up to two years post-surgery revealed significantly higher scores in the cell-treated group [66].

Concerns Regarding Current Clinical-Treatment Procedures There are several common limitations, of which at least one applies to each clinical study listed. First and most importantly, most studies lack quantitative and histological evidence. Second, many studies are level IV studies, and therefore either no matched control group is included or the number of cases is very small. In addition, the follow-up period is often too short and it would be desirable to have more than one arthroscopy observation during the follow-up time. The latter, of course, is associated with ethical concerns. Third, almost every study used different treatment procedures, i.e. intraarticular injection versus implantation or including MSCs from different tissue sources, and thus they can be regarded only as pilot studies.

\section{Conclusions}

Only a few published pre-clinical, large animal, and clinical studies of MSC-based treatment of OA-induced chondral and osteochondral lesions are available (Fig. 1). Most pre-clinical studies using large-animal models provide MSC-based treatment procedures for isolated focal chondral and osteochondral lesions encompassed by healthy cartilage and bone. Furthermore, they do not address special requirements for cell-based strategies adapted to treat $\mathrm{OA}$-induced degenerative large cartilage defects. In addition, all studies used expanded BMSCs, requiring a two-step treatment, and none used 
BMC, which have important clinical implications because they can be applied without leaving the operating theatre, circumventing regulatory obstacles. Clinical studies are mostly uncontrolled case reports including only a few patients, and detailed molecular and histological analyses of repair tissue are not feasible for ethical reasons. In addition, study procedures, follow-up times, cell sources, and biomaterials differ greatly among the studies, thereby preventing generalized conclusions on clinical and functional outcomes. However, preliminary results of pre-clinical and clinical studies are promising. In general, after cell-based therapy-irrespective of cell type - clinical and functional scores are clearly improved and defects are filled with newly formed cartilage-like tissue, sometimes even with hyaline-like characteristics.

Acknowledgments This work was supported by a DFG grant to SG (GR 1301/9-1).

\section{Compliance with Ethics Guidelines}

Conflict of Interest Susanne Grässel and Julia Lorenz declare the receipt of grants from Deutsche Forschungsgemeinschaft (DFG).

Human and Animal Rights and Informed Consent This article does not contain any studies with human or animal subjects performed by any of the authors.

Open Access This article is distributed under the terms of the Creative Commons Attribution License which permits any use, distribution, and reproduction in any medium, provided the original author(s) and the source are credited.

\section{References}

Papers of particular interest, published recently, have been highlighted as:

- Of importance

1. Pitsillides AA, Beier F. Cartilage biology in osteoarthritis-lessons from developmental biology. Nat Rev Rheumatol. 2011;7(11):65463.

2. van den Berg WB. Osteoarthritis year 2010 in review: pathomechanisms. Osteoarthr Cartil. 2011;19(4):338-41.

3. Dreier R. Hypertrophic differentiation of chondrocytes in osteoarthritis: the developmental aspect of degenerative joint disorders. Arthritis Res Ther. 2010;12(5):216

4. Goldring MB, Marcu KB. Cartilage homeostasis in health and rheumatic diseases. Arthritis Res Ther. 2009;11(3):224.

5. Mitchell N, Shepard N. Effect of patellar shaving in the rabbit. J Orthop Res. 1987;5(3):388-92.

6. Hjelle K, Solheim E, Strand T, et al. Articular cartilage defects in 1, 000 knee arthroscopies. Arthroscopy. 2002;18(7):730-4.

7. Clouet J, Vinatier C, Merceron C, et al. From osteoarthritis treatments to future regenerative therapies for cartilage. Drug Discov Today. 2009;14(19-20):913-25.
8. Buckwalter JA, Brown TD. Joint injury, repair, and remodeling: roles in post-traumatic osteoarthritis. Clin Orthop Relat Res. 2004;423:7-16.

9. Buckwalter JA, Saltzman C, Brown T. The impact of osteoarthritis: implications for research. Clin Orthop Relat Res. 2004(427 Suppl): S6-15.

10. Steinert AF, Ghivizzani SC, Rethwilm A, et al. Major biological obstacles for persistent cell-based regeneration of articular cartilage. Arthritis Res Ther. 2007;9(3):213.

11. Steinert AF, Rackwitz L, Gilbert F, et al. Concise review: the clinical application of mesenchymal stem cells for musculoskeletal regeneration: current status and perspectives. Stem Cells Transl Med. 2012;1(3):237-47. This is an up to date review on clinical application of MSCs for bone, cartilage, and tendon regeneration. It discusses current clinical studies on cell-based therapies of nonunions, osteonecrosis of the hip, bone tumors, articular cartilage lesions, and ligament and tendon ruptures.

12. Frisbie DD, Cross MW, McIlwraith CW. A comparative study of articular cartilage thickness in the stifle of animal species used in human pre-clinical studies compared to articular cartilage thickness in the human knee. Vet Comp Orthop Traumatol VCOT. 2006;19(3):142-6.

13. Bouwmeester PS, Kuijer R, Homminga GN, et al. A retrospective analysis of two independent prospective cartilage repair studies: autogenous perichondrial grafting versus subchondral drilling 10 years post-surgery. J Orthop Res Off Publ Orthop Res Soc. 2002;20(2):267-73.

14. Ahern BJ, Parvizi J, Boston R, et al. Preclinical animal models in single site cartilage defect testing: a systematic review. Osteoarthr Cartil OARS Osteoarthr Res Soc. 2009;17(6):705-13.

15. Mrugala D, Bony $\mathrm{C}$, Neves $\mathrm{N}$, et al. Phenotypic and functional characterisation of ovine mesenchymal stem cells: application to a cartilage defect model. Ann Rheum Dis. 2008;67(3):288-95.

16. Zscharnack M, Hepp P, Richter R, et al. Repair of chronic osteochondral defects using predifferentiated mesenchymal stem cells in an ovine model. Am J Sports Med. 2010;38(9): 1857-69.

17. Marquass B, Schulz R, Hepp P, et al. Matrix-associated implantation of predifferentiated mesenchymal stem cells versus articular chondrocytes: in vivo results of cartilage repair after 1 year. Am J Sports Med. 2011;39(7):1401-12. This pre-clinical study compares the regenerative capacities of pre-differentiated BMSCs versus chondrocytes implanted into osteochondral lesions in a sheep model. BMSC performance was superior to chondrocytes with respect to O'Driscoll and ICRS scores until 1-year follow up.

18. Marquass B, Somerson JS, Hepp P, et al. A novel MSC-seeded triphasic construct for the repair of osteochondral defects. J Orthop Res. 2010;28(12):1586-99.

19. Guo X, Wang C, Zhang Y, et al. Repair of large articular cartilage defects with implants of autologous mesenchymal stem cells seeded into beta-tricalcium phosphate in a sheep model. Tissue Eng. 2004;10(11-12):1818-29.

20. Frosch KH, Drengk A, Krause P, et al. Stem cell-coated titanium implants for the partial joint resurfacing of the knee. Biomaterials. 2006;27(12):2542-9.

21. Steck E, Fischer J, Lorenz H, et al. Mesenchymal stem cell differentiation in an experimental cartilage defect: restriction of hypertrophy to bone-close neocartilage. Stem Cells Dev. 2009;18(7): 969-78.

22. Zhou G, Liu W, Cui L, et al. Repair of porcine articular osteochondral defects in non-weightbearing areas with autologous bone marrow stromal cells. Tissue Eng. 2006;12(11): 3209-21.

23. Ho ST, Hutmacher DW, Ekaputra AK, et al. The evaluation of a biphasic osteochondral implant coupled with an electrospun membrane in a large animal model. Tissue Eng A. 2010;16(4):1123-41. 
24. Chang CH, Kuo TF, Lin FH, et al. Tissue engineering-based cartilage repair with mesenchymal stem cells in a porcine model. J Orthop Res Off Publ Orthop Res Soc. 2011;29(12):1874-80. This study compared repair capacity of pre-differentiated with undifferentiated BMSCs implanted in osteochondral defects in minipigs. The quality of the repair tissue was inferior and no difference between groups was observed.

25. Wilke MM, Nydam DV, Nixon AJ. Enhanced early chondrogenesis in articular defects following arthroscopic mesenchymal stem cell implantation in an equine model. J Orthop Res Off Publ Orthop Res Soc. 2007;25(7):913-25.

26. Seo JP, Tanabe T, Tsuzuki N, et al. Effects of bilayer gelatin/betatricalcium phosphate sponges loaded with mesenchymal stem cells, chondrocytes, bone morphogenetic protein-2, and platelet rich plasma on osteochondral defects of the talus in horses. Res Vet Sci. 2013;95(3):1210-6. This study analyzed repair the capacity of BMSCs loaded onto biphasic sponge scaffolds and implanted in osteochondral defects in horses. After 4 months, the test group had significantly higher radiographic, QCT, macroscopic, and histological scores than the control group with biphasic sponge without cells and growth factor.

27. Lu Y, Hayashi K, Hecht P, et al. The effect of monopolar radiofrequency energy on partial-thickness defects of articular cartilage. Arthrosc J Arthrosc Relat Surg Off Publ Arthrosc Assoc N Am Int Arthrosc Assoc. 2000;16(5):527-36.

28. O'Driscoll SW, Marx RG, Beaton DE, et al. Validation of a simple histological-histochemical cartilage scoring system. Tissue Eng. 2001;7(3):313-20.

29. Mainil-Varlet P, Aigner T, Brittberg M, et al. Histological assessment of cartilage repair: a report by the Histology Endpoint Committee of the International Cartilage Repair Society (ICRS). J Bone Joint Surg Am Vol. 2003;85-A Suppl 2:45-57.

30. Chiang H, Kuo TF, Tsai CC, et al. Repair of porcine articular cartilage defect with autologous chondrocyte transplantation. J Orthop Res Off Publ Orthop Res Soc. 2005;23(3):584-93.

31. Hembry RM, Dyce J, Driesang I, et al. Immunolocalization of matrix metalloproteinases in partial-thickness defects in pig articular cartilage. A preliminary report. J Bone Joint Surg Am Vol. 2001;83-A(6):826-38.

32. Pineda S, Pollack A, Stevenson S, et al. A semiquantitative scale for histologic grading of articular cartilage repair. Acta Anat. 1992;143(4):335-40.

33. Bryant SJ, Anseth KS. The effects of scaffold thickness on tissue engineered cartilage in photocrosslinked poly(ethylene oxide) hydrogels. Biomaterials. 2001;22(6):619-26.

34. Noth U, Steinert AF, Tuan RS. Technology insight: adult mesenchymal stem cells for osteoarthritis therapy. Nat Clin Pract Rheumatol. 2008;4(7):371-80.

35. Grassel S, Anders S. Cell-based therapy options for osteochondral defects. Autologous mesenchymal stem cells compared to autologous chondrocytes. Orthopade. 2012;41(5):415-28. quiz 429-430.

36. Steinwachs MR, Guggi T, Kreuz PC. Marrow stimulation techniques. Injury. 2008;39 Suppl 1:S26-31.

37. Richter W. Mesenchymal stem cells and cartilage in situ regeneration. J Intern Med. 2009;266(4):390-405.

38. Blevins FT, Steadman JR, Rodrigo JJ, et al. Treatment of articular cartilage defects in athletes: an analysis of functional outcome and lesion appearance. Orthopedics. 1998;21(7):761-7. discussion $767-768$.

39. Steadman JR, Briggs KK, Rodrigo JJ, et al. Outcomes of microfracture for traumatic chondral defects of the knee: average 11-year follow-up. Arthroscopy. 2003;19(5):477-84.

40. Richter W. Cell-based cartilage repair: illusion or solution for osteoarthritis. Curr Opin Rheumatol. 2007;19(5):451-6.

41. Filardo G, Madry H, Jelic M, et al. Mesenchymal stem cells for the treatment of cartilage lesions: from preclinical findings to clinical application in orthopaedics. Knee Surg Sports Traumatol Arthrosc Off J ESSKA. 2013;21(8):1717-29. This is an up-to-date systematic review on clinical studies using MSC treatment strategies for cartilage defect regeneration.

42. Gigante A, Calcagno S, Cecconi S, et al. Use of collagen scaffold and autologous bone marrow concentrate as a one-step cartilage repair in the knee: histological results of second-look biopsies at 1 year follow-up. Int J Immunopathol Pharmacol. 2011;24(1 Suppl 2):69-72.

43. Gigante A, Cecconi S, Calcagno S, et al. Arthroscopic knee cartilage repair with covered microfracture and bone marrow concentrate. Arthrosc Tech. 2012;1(2):e175-80.

44. Enea D, Cecconi S, Calcagno S, et al. Single-stage cartilage repair in the knee with microfracture covered with a resorbable polymerbased matrix and autologous bone marrow concentrate. Knee. 2013;20(6):562-9. This case series describes treatment of traumainduced focal condylar knee lesions with a combination of MFX, biomaterial, and bone marrow concentrate. They report significant improvement of several clinical scores and complete defect filling.

45. Giannini S, Buda R, Vannini F, et al. One-step bone marrowderived cell transplantation in talar osteochondral lesions. Clin Orthop Relat Res. 2009;467(12):3307-20.

46. Skowronski J, Skowronski R, Rutka M. Large cartilage lesions of the knee treated with bone marrow concentrate and collagen membrane-results. Ortop Traumatol Rehabil. 2013;15(1):69-76.

47. Giannini S, Buda R, Cavallo M, et al. Cartilage repair evolution in post-traumatic osteochondral lesions of the talus: from open field autologous chondrocyte to bone-marrow-derived cells transplantation. Injury. 2010;41(11):1196-203.

48. Wakitani S, Mitsuoka T, Nakamura N, et al. Autologous bone marrow stromal cell transplantation for repair of full-thickness articular cartilage defects in human patellae: two case reports. Cell Transplant. 2004;13(5):595-600.

49. Kuroda R, Ishida K, Matsumoto $T$, et al. Treatment of a fullthickness articular cartilage defect in the femoral condyle of an athlete with autologous bone-marrow stromal cells. Osteoarthr Cartil OARS Osteoarthr Res Soc. 2007;15(2): 226-31.

50. Wakitani S, Nawata M, Tensho K, et al. Repair of articular cartilage defects in the patello-femoral joint with autologous bone marrow mesenchymal cell transplantation: three case reports involving nine defects in five knees. J Tissue Eng Regen Med. 2007;1(1):74-9.

51. Haleem AM, Singergy AA, Sabry D, et al. The clinical use of human culture-expanded autologous bone marrow mesenchymal stem cells transplanted on platelet-rich fibrin glue in the treatment of articular cartilage defects: a pilot study and preliminary results. Cartilage. 2010;1(4):253-61.

52. Nejadnik H, Hui JH, Feng Choong EP, et al. Autologous bone marrow-derived mesenchymal stem cells versus autologous chondrocyte implantation: an observational cohort study. Am J Sports Med. 2010;38(6):1110-6.

53. Varma HS, Dadarya B, Vidyarthi A. The new avenues in the management of osteo-arthritis of knee-stem cells. J Indian Med Assoc. 2010;108(9):583-5.

54. Kim JD, Lee GW, Jung GH, et al. Clinical outcome of autologous bone marrow aspirates concentrate (BMAC) injection in degenerative arthritis of the knee. Eur J Orthop Surg Traumatol Orthop Traumatol. 2014. This group combined intraarticular injection of autologous BMC with adipose tissue (BMAC) into knees of 41 patients with radiographically assessed OA. BMAC injection significantly improved both knee pain and function in all patients.

55. Centeno CJ, Busse D, Kisiday J, et al. Increased knee cartilage volume in degenerative joint disease using percutaneously 
implanted, autologous mesenchymal stem cells. Pain Physician. 2008;11(3):343-53.

56. Centeno CJ, Schultz JR, Cheever M, et al. Safety and complications reporting on the re-implantation of culture-expanded mesenchymal stem cells using autologous platelet lysate technique. Curr Stem Cell Res Ther. 2010;5(1):81-93.

57. Centeno CJ, Schultz JR, Cheever M, et al. Safety and complications reporting update on the re-implantation of culture-expanded mesenchymal stem cells using autologous platelet lysate technique. Curr Stem Cell Res Ther. 2011;6(4):368-78.

58. Emadedin M, Aghdami N, Taghiyar L, et al. Intra-articular injection of autologous mesenchymal stem cells in six patients with knee osteoarthritis. Arch Iran Med. 2012;15(7):422-8. This is the first group who injected expanded BMSCs into knee joints of $O A$ patients.

59. Davatchi F, Abdollahi BS, Mohyeddin M, et al. Mesenchymal stem cell therapy for knee osteoarthritis. Preliminary report of four patients. Int J Rheum Dis. 2011;14(2):211-5.

60. Pak J. Regeneration of human bones in hip osteonecrosis and human cartilage in knee osteoarthritis with autologous adipose-tissuederived stem cells: a case series. J Med Case Rep. 2011;5:296. This is the first group who injected concentrated (not expanded) ADSCs in combination with PRP into knee joints of OA patients.
61. Koh YG, Choi YJ. Infrapatellar fat pad-derived mesenchymal stem cell therapy for knee osteoarthritis. Knee. 2012;19(6):902-7.

62. Koh YG, Jo SB, Kwon OR, et al. Mesenchymal stem cell injections improve symptoms of knee osteoarthritis. Arthroscopy. 2013;29(4): 748-55.

63. Koh YG, Choi YJ, Kwon SK, et al. Clinical results and second-look arthroscopic findings after treatment with adipose-derived stem cells for knee osteoarthritis. Knee Surg Sports Traumatol Arthrosc Off J ESSKA. 2013.

64. Wakitani S, Imoto K, Yamamoto T, et al. Human autologous culture expanded bone marrow mesenchymal cell transplantation for repair of cartilage defects in osteoarthritic knees. Osteoarthr Cartil. 2002;10(3):199-206.

65. Koh YG, Choi YJ, Kwon OR, et al. Second-look arthroscopic evaluation of cartilage lesions after mesenchymal stem cell implantation in osteoarthritic knees. Am J Sports Med. 2014. This study implanted ASDCs without expansion into isolated full-thickness cartilage lesions of $O A$ patients.

66. Wong KL, Lee KB, Tai BC, et al. Injectable cultured bone marrowderived mesenchymal stem cells in varus knees with cartilage defects undergoing high tibial osteotomy: a prospective, randomized controlled clinical trial with 2 years' follow-up. Arthroscopy. 2013;29(12):2020-8. 